\title{
Systemic air embolism after percutaneous computed tomography-guided lung biopsy due to a kink in the coaxial biopsy system: a case report
}

Hsu-Chao Chang ${ }^{1}$ and Mei-Chen Yang ${ }^{2,3^{*}}$

\begin{abstract}
Background: Systemic air embolism is a rare but potentially life-threatening complication of percutaneous computed tomography (CT)-guided lung biopsy. The incidence might be underestimated because of failure to diagnose this adverse event in asymptomatic patients; early recognition is difficult.

Case presentation: We report the case of a 73-year-old man with systemic air embolism, a complication of percutaneous CT-guided lung biopsy, due to a kink in the coaxial biopsy system. Serial post-procedure CT scans demonstrated the causal relationship.

Conclusions: Sequential post-biopsy CT scans demonstrated a causal relationship between this systemic air embolism and percutaneous biopsy, and allowed the radiologist to track the course of the emboli and their resolution. Awareness of air entry via the introducer needle and an early post-biopsy CT scan are crucial for early detection of systemic air embolism. If air embolism occurs in an asymptomatic patient, we recommend performing a delayed chest CT scan to follow the air's course.
\end{abstract}

Keywords: Complication, Lung mass, Chest imaging

\section{Background}

Systemic air embolism after percutaneous computed tomography $(\mathrm{CT})$-guided lung biopsy is very rare. The estimated incidence is $0.061-0.21 \%$ [1, 2], but could be underestimated because of failure to diagnose this adverse event in asymptomatic patients [3]. For example, in a study published in 2012, Freund et al. found that the incidence of radiologically proven systemic air embolism was $3.8 \%$, whereas the incidence of clinically apparent systemic air embolism was $0.49 \%$ [4]. Thus, the incidence of post-biopsy systemic air embolism might be higher than is estimated, and sequential CT scans of the entire thorax instead of only the biopsy area might be worth performing to provide a definitive diagnosis of

\footnotetext{
* Correspondence: mimimai3461@gmail.com

${ }^{2}$ School of Medicine, Tzu Chi University, Hualien, Taiwan

${ }^{3}$ Division of Pulmonary Medicine, Department of Internal Medicine, Taipei Tzu-Chi Hospital, Buddhist Tzu-Chi Medical Foundation, No. 289, Jianguo Rd, Xindian Dist, New Taipei City 23143, Taiwan

Full list of author information is available at the end of the article
}

systemic air embolism [5]. Generally, systemic air embolism is thought to occur via two mechanisms. First, if the tip of the biopsy needle is lodged in a pulmonary vein and the inner stylet is removed, air embolism occurs during rapid inspiration when atmospheric pressure exceeds pulmonary venous pressure. Second, when the needle simultaneously traverses an air-containing space and adjacent pulmonary vein, a fistula can occur, resulting in air entering the pulmonary vein when the alveolar pressure is greater than the pulmonary venous pressure [6]. Air can enter via a fistula tract, connecting an aircontaining space to a pulmonary vein when alveolar pressure is high, for example, during coughing. To prevent air embolism, the introducer needle should always be occluded by the inner stylet, saline drops, or the operator's finger. Moreover, the patient should be instructed to avoid breathing deeply or coughing forcefully during the procedure $[7,8]$. It is believed that, following lung biopsy and needle withdrawal, a large 
number of alveolar-venous or bronchial-venous fistulas can develop along the needle's trajectory. During vascular injury, the walls of small vessels are usually retracted; spontaneous adhesion occurs, forming a seal. When a patient exhibits any symptoms suggestive of air embolism during the lung biopsy procedure, the needle should immediately be removed $[8,9]$. The clinical manifestations of air embolism vary, depending on the exact location of the arterial embolus and the volume of air disseminated into the vessels. Once air enters the systemic circulation, it will enter the respective arterial end beds [1, 8]. Functional end arteries can be occluded by even a small volume of air. Only $2 \mathrm{~mL}$ of air injected directly into the cerebral circulation is enough to be fatal, and $0.5-1.0 \mathrm{~mL}$ of air injected into a coronary artery can lead to cardiac arrest [10]. Initial management includes immediate administration of $100 \%$ oxygen and placing the patient in a slight Trendelenburg position [7]. Early hyperbaric oxygen therapy is recommended for patients with cerebral air embolism [5].

Previously reported cases have only demonstrated imaging findings as existing air emboli in post-procedure CT scans. Imaging findings at different times, and the use of those findings to track the course and resolution of air emboli, have not been described. We report a case of systemic air embolism after CT-guided biopsy due to a kink in the coaxial biopsy system, and show the course followed by the emboli, demonstrated on sequential CT scans.

\section{Case presentation}

A 73-year-old man with pneumoconiosis, chronic obstructive pulmonary disease, and hypertension who had previously had a cerebrovascular accident and had undergone surgery for gastric cancer ten years earlier, was brought to our emergency department with a one-day history of acute-onset tremor and leg weakness. The chest radiograph and chest CT scan showed a large mass in the right middle lobe (RML) that was out of keeping with his pneumoconiosis. Lung malignancy was of concern; hence, a CT-guided percutaneous lung biopsy was suggested and was performed after obtaining informed consent.

A coaxial biopsy system with a 19-gauge introducer needle and a 20-gauge core biopsy needle (length, $17 \mathrm{~cm}$; Temno, CareFusion, France) was selected for this procedure. Once the introducer needle had reached the lesion, the internal stylet was removed and the core biopsy needle was inserted to procure a specimen. However, after obtaining the first specimen, we were unable to withdraw the core biopsy needle because of a kink. Several attempts to withdraw it resulted in it being pushed forward and kinked again; finally, it was forcibly removed. The core biopsy needle was found to be angulated proximally. Thereupon, free air sprang up from the introducer needle. Hence, the stylet was reinserted and the introducer needle was immediately removed. After withdrawal of the instrument, the patient coughed forcefully. An immediate post-biopsy CT scan was performed; it demonstrated free air with flow-related motion artifact within the RML mass, the left atrium, and the right pulmonary vein (Fig. 1a and b). A follow-up CT scan of the entire thorax demonstrated air collections in the ascending aorta and the right coronary artery (Fig. 1c and d). Because the patient had no symptoms or signs of acute

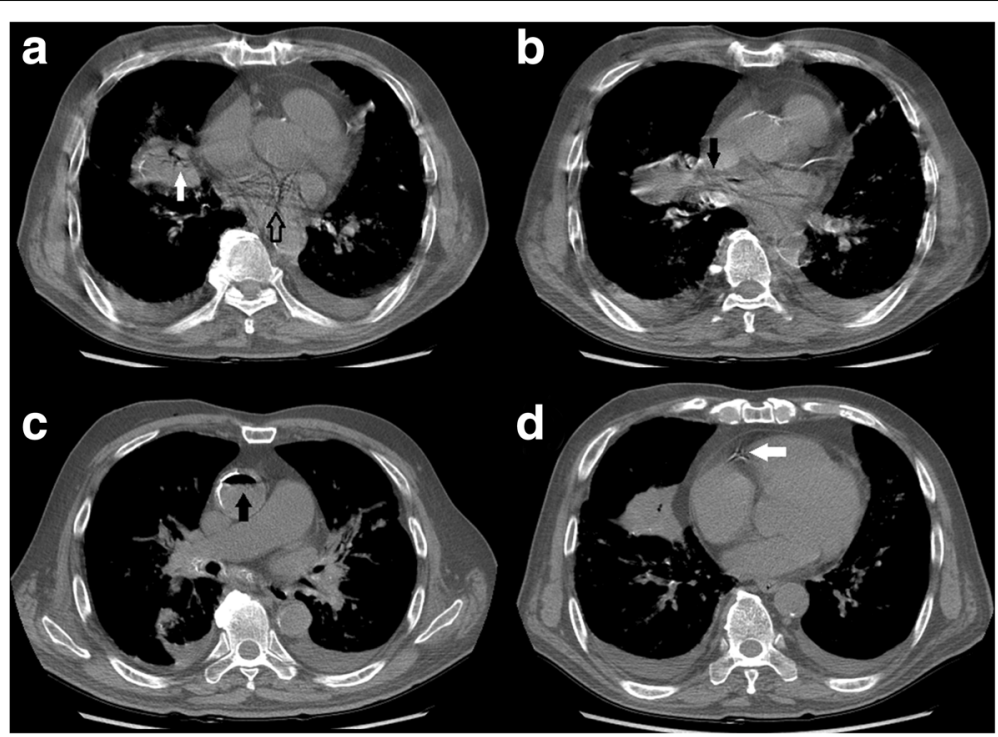

Fig. 1 Series of computed tomography scans demonstrating the movement of the air emboli. Immediate post-procedure computed tomography scans demonstrated free air with flow-related motion artifact in a the right middle lobe mass (white arrow), and the left atrium (open arrow), and b the right pulmonary vein (black arrow). After $50 \mathrm{~s}$, a follow-up computed tomography scan demonstrated air collections in c the ascending aorta (black arrow) and $\mathbf{d}$ right coronary artery (white arrow) 
stroke or myocardial infarction, he was kept in the CT room and oxygen was administered. Ten minutes later, a delayed chest $\mathrm{CT}$ scan demonstrated no air collections in the aorta or left heart, and total resolution of the air embolus in the right coronary artery (Fig. 2). The patient's condition was stable and an electrocardiogram showed no ST-segment elevation. He was thus transferred to the intensive care unit for close monitoring.

During his stay in the intensive care unit, neither air embolism nor new neurologic deficits developed, and his troponin I and CK-MB levels were normal. Five days later, brain magnetic resonance imaging was performed; it showed no evidence of acute infarction. The patient was transferred to the ward and was discharged days later in a stable condition. Table 1 shows the timeline of course of events.

\section{Discussion}

As demonstrated in this report, post-biopsy sequential CT scans can show the causal relationship between systemic air embolism and percutaneous biopsy, and can track the course and resolution of such emboli. The serial CT scans performed on our patient showed air with flow-related motion artifact in the lung mass, pulmonary vein, left heart, and then in the aorta and coronary artery. Later, the CT scans showed resolution of the air collections in the aorta and left heart, and almost total resolution of the air embolus in the coronary artery. Such evolution occurred quickly. Had the radiologists been unaware of this complication, CT scans would have been delayed and the systemic air emboli would either not have been detected or the diagnosis would have been delayed.

How can one avoid causing an angulation deformity in the core biopsy needle, thereby causing it to kink and get

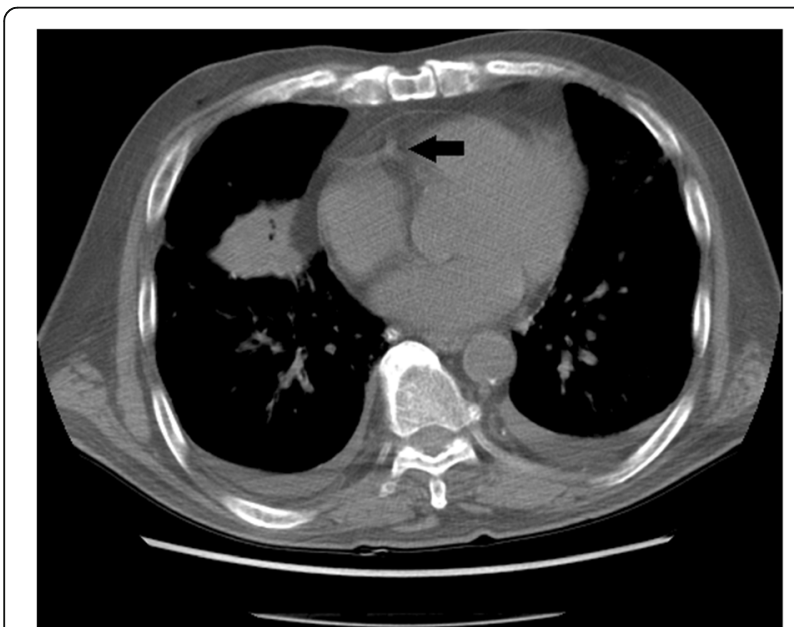

Fig. 2 Delayed chest computed tomography scan, performed after $10 \mathrm{~min}$, demonstrating total resolution of the air embolus in the right coronary artery
Table 1 Timeline of events

\begin{tabular}{ll}
\hline Date & Event \\
\hline 6 Nov & Admission for CT-guided lung biopsy \\
7 Nov & $\begin{array}{l}\text { Systemic air embolism after percutaneous CT-guided } \\
\text { lung biopsy due to a kink in the coaxial biopsy system }\end{array}$ \\
$7 \mathrm{Nov}$ & $\begin{array}{l}\text { No ST-segment elevation on electrocardiogram; } \\
\text { normal troponin I and CK-MB levels }\end{array}$ \\
$12 \mathrm{Nov}$ & $\begin{array}{l}\text { No acute infarct on brain magnetic resonance imaging } \\
17 \mathrm{Nov}\end{array}$ \\
\hline
\end{tabular}

stuck in the introducer needle? Although blood clots can cause the biopsy needle to adhere to the introducer needle, especially when a long instrument is used, such adherence can be managed by flushing the introducer needle. After several experiments, we determined that the core biopsy needle deformity had occurred because the needle had been inappropriately removed from its packaging, resulting in angulation at the fulcrum where pressure was applied (Fig. 3). If the needle is inadvertently kinked, the biopsy set should be instantly discarded to prevent such severe complications as occurred in this case.

In the case presented, the air definitely came from the atmosphere because we witnessed air being drawn into the introducer needle. Moreover, the to-and-fro motion of the kinked core biopsy needle in the introducer needle may have evacuated the mass. Delayed reinsertion of the introducer needle's stylet after forcibly removing the kinked biopsy needle also played an important role. Early awareness of air being drawn into the introducer needle is very important. An immediate series of CT scans to trace the air's course is crucial for early detection of systemic air embolism.

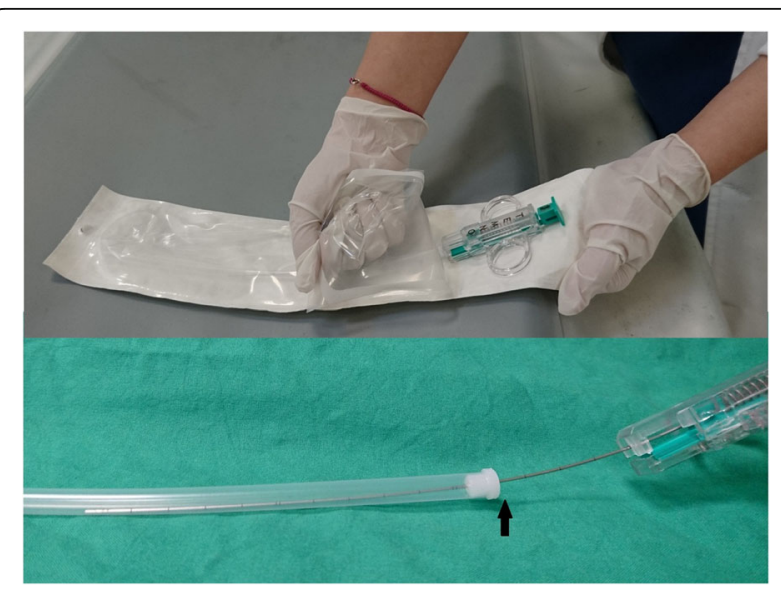

Fig. 3 Inappropriate removal of the core biopsy needle from its packaging. The upper image demonstrates an inappropriate method of removing the core biopsy needle from its packaging, resulting in angulation at the fulcrum where pressure was applied (lower image, black arrow) 
Initial management includes immediate administration of $100 \%$ oxygen, and placing the patient in a slight Trendelenburg position [7]. Early hyperbaric oxygen therapy is recommended for patients with cerebral air embolism [5]. However, there is no standard protocol for managing asymptomatic patients; we recommend performing delayed post-procedure CT scans to evaluate the air's course.

Two major events need to be considered: Embolic stroke and myocardial infarction. Clinical features that support the diagnosis of cardioembolic stroke include the sudden onset of neurological deficits, an interval from onset to maximal deficit of $<5 \mathrm{~min}$, and rapid regression of symptoms [11]. The symptoms of cerebral arterial air embolism develop suddenly. The clinical presentation is determined by the absolute amount of air and the intracranial vessel territories affected by the embolic event [12]. If delayed post-procedure CT scans show no more air in the left heart or in the thoracic aorta, there is no further concern of cerebral air embolism occurring. However, the symptoms of myocardial infarction may be atypical and silent $[13,14]$. The electrocardiogram demonstrates ST-segment elevation rapidly (within $30 \mathrm{~s}$ ) after manual transient occlusion of a coronary artery [15]. The incidence of acute non-ST elevation myocardial infarction may be higher than acute ST elevation myocardial infarction [16]. In our daily practice, a post-biopsy CT scan is performed routinely in every patient to evaluate complications. If air embolism is detected in a routine post-biopsy CT scan, another delayed CT scan is recommended to follow the air's course in an asymptomatic patient. If this delayed CT scan does not demonstrate air emboli in the coronary artery, left heart, or thoracic aorta, either no air entry occurred or the coronary artery has recanalized. In this patient, we evaluated the coronary arteries in the delayed post-procedure CT scan images to ensure that the air embolism had completely resolved. However, if the air embolism persists or progresses, the patient should be intensively monitored (e.g. symptom and electrocardiogram monitoring, and serial measurement of cardiac enzymes).

\section{Conclusion}

Sequential post-biopsy CT scans of this patient demonstrated the causal relationship between systemic air embolism and percutaneous biopsy, and allowed the radiologist to track the course and resolution of the emboli. Awareness of air entry via the introducer needle and an early post-biopsy CT scan are crucial for early detection of systemic air embolism. We recommend that a delayed chest CT scans be performed to follow the air's course when air embolism is detected in an asymptomatic patient.

Abbreviations

$\mathrm{CT}$ : Computed tomography; RML: Right middle lobe

\section{Acknowledgements}

We would like to thank Editage (www.editage.com) for English language editing.

\section{Funding}

This case report was supported by a grant from Taipei Tzu-Chi Hospital, Buddhist Tzu-Chi Medical Foundation (TCRD-TPE-106-RT-1). The funders played no role in the design of the study and collection, analysis, and interpretation of data and in writing the manuscript.

\section{Availability of data and materials}

All data generated or analyzed during this study are included in this published article.

\section{Authors' contributions}

HCC collected the data and references, followed up the patient, performed the literature review, and drafted the manuscript. MCY critically revised the manuscript and assisted in the literature review. Both authors have read and approved the final manuscript.

\section{Ethics approval and consent to participate}

This report was approved by the Institutional Review Board of Taipei Tzu Chi Hospital, Buddhist Tzu Chi Medical Foundation (protocol No.: 06-CR03-040) and adheres to the ethical standards of the 1964 Helsinki Declaration. Written informed consent for publication was provided by the daughter-in-law of the patient since patient had died due to pneumonia 2 years after this admission, prior to writing this report.

\section{Consent for publication}

Written informed consent for publication of the case report was obtained from the patient's daughter-in-law.

\section{Competing interests}

The authors declare that they have no competing interests.

\section{Publisher's Note}

Springer Nature remains neutral with regard to jurisdictional claims in published maps and institutional affiliations.

\section{Author details}

${ }^{1}$ Department of Radiology, Taipei Tzu-Chi Hospital, Buddhist Tzu-Chi Medical Foundation, No. 289, Jianguo Rd, Xindian Dist, New Taipei City 23143,

Taiwan. ${ }^{2}$ School of Medicine, Tzu Chi University, Hualien, Taiwan. ${ }^{3}$ Division of Pulmonary Medicine, Department of Internal Medicine, Taipei Tzu-Chi Hospital, Buddhist Tzu-Chi Medical Foundation, No. 289, Jianguo Rd, Xindian Dist, New Taipei City 23143, Taiwan.

Received: 25 July 2017 Accepted: 17 January 2018

Published online: 27 January 2018

\section{References}

1. Tomiyama N, Yasuhara Y, Nakajima Y, Adachi S, Arai Y, Kusumoto M, et al. CT-guided needle biopsy of lung lesions: a survey of severe complication based on 9783 biopsies in Japan. Eur J Radiol. 2006;2006(59):60-4.

2. Ibukuro K, Tanaka R, Takeguchi T, Fukuda H, Abe S, Tobe K. Air embolism and needle track implantation complicating CT-guided percutaneous thoracic biopsy: single-institution experience. AJR Am J Roentgenol. 2009; 2009(193):W430-6.

3. Ghafoori M, Varedi P. Systemic air embolism after percutaneous transthorasic needle biopsy of the lung. Emerg Radiol. 2008;15:353-6.

4. Freund MC, Petersen J, Goder KC, Bunse T, Wiedermann F, Glodny B. Systemic air embolism during percutaneous core needle biopsy of the lung: frequency and risk factors. BMC Pulm Med. 2012;12:2.

5. Blanc P, Boussuges A, Henriette K, Sainty JM, Deleflie M. latrogenic cerebral air embolism: importance of an early hyperbaric oxygenation. Intensive Care Med. 2002;28:559-63.

6. Wu CC, Maher MM, Shepard JA. Complications of CT-guided percutaneous needle biopsy of the chest: prevention and management. AJR Am J Roentgenol. 2011;196:W678-82.

7. Bou-Assaly W, Pernicano P, Hoeffner E. Systemic air embolism after transthoracic lung biopsy: a case report and review of literature. World J Radiol. 2010;2:193-6. 
8. Hiraki T, Fujiwara H, Sakurai J, Iguchi T, Gobara H, Tajiri N, et al. Nonfatal systemic air embolism complicating percutaneous CT-guided transthoracic needle biopsy: four cases from a single institution. Chest. 2007;132:684-90.

9. Kau T, Rabitsch E, Celedin S, Habernig SM, Weber JR, Hausegger KA. When coughing can cause stroke-a case-based update on cerebral air embolism complicating biopsy of the lung. Cardiovasc Intervent Radiol. 2008;31:848-53.

10. Ho AM, Ling E. Systemic air embolism after lung trauma. Anesthesiology. 1999:90:564-75.

11. Arboix A, Alio J. Cardioembolic stroke: clinical features, specific cardiac disorders and prognosis. Curr Cardiol Rev. 2010;6:150-61.

12. Muth CM, Shank ES. Gas embolism. N Engl J Med. 2000;342:476-82.

13. Valensi $P$, Lorgis $L$, Cottin Y. Prevalence, incidence, predictive factors and prognosis of silent myocardial infarction: a review of the literature. Arch Cardiovasc Dis. 2011;104:178-88.

14. Fujino M, Ishihara M, Ogawa H, Nakao K, Yasuda S, Noguchi T, et al. Impact of symptom presentation on in-hospital outcomes in patients with acute myocardial infarction. J Cardiol. 2017;70:29-34.

15. Zalewski A, Goldberg S, Dervan JP, Slysh S, Maroko PR. Myocardial protection during transient coronary artery occlusion in man: beneficial effects of regional beta-adrenergic blockade. Circulation. 1986;73:734-9.

16. McManus DD, Gore J, Yarzebski J, Spencer F, Lessard D, Goldberg RJ. Recent trends in the incidence, treatment, and outcomes of patients with STEMI and NSTEMI. Am J Med. 2011;124:40-7.

\section{Submit your next manuscript to BioMed Central and we will help you at every step:}

- We accept pre-submission inquiries

- Our selector tool helps you to find the most relevant journal

- We provide round the clock customer support

- Convenient online submission

- Thorough peer review

- Inclusion in PubMed and all major indexing services

- Maximum visibility for your research

Submit your manuscript at www.biomedcentral.com/submit 